\title{
Stark width and shift parameter predictions and regularities of Sn II
}

\author{
C Colón \\ A Alonso Medina \\ C Rivero \\ F Fernández
}

\begin{abstract}
Semi-classical approximate values of the Stark parameters for several lines of Sn II are reported in this paper. They were calculated using a set of wavefunctions obtained from Hartree-Fock relativistic calculations. Stark widths for 80 lines of Sn II arising from ns ${ }^{2} \mathrm{~S}_{1 / 2}$, $n{ }^{2} \mathrm{P}_{1 / 2,3 / 2}$, nd ${ }^{2} \mathrm{D}_{3 / 2,5 / 2}$ and $n{ }^{2} \mathrm{~F}_{5 / 2,7 / 2}$ levels of $\mathrm{Sn}$ II have been calculated in this way. Stark widths are presented as a function of temperature for an electron density of $1 \mathbf{0}^{17} \mathrm{~cm}^{-3}$. Our results are compared with available experimental data. Stark shifts of five lines with experimental value in the bibliography have been studied normalized to an electron density of $10^{17} \mathrm{~cm}^{-3}$. Common regularities for the Stark width of several lines of singly ionized tin have been discussed.
\end{abstract}

\section{Introduction}

Measurements of Stark-broadening parameters provide means for diagnosis of laboratory and astrophysical plasmas. Stark-broadening data for a large number of transitions in many atomic and ionic spectra are needed for the mod elling of stellar plasma, the investigation of its physical properties and abundance determinations. When calculating opacities of large-scale plasmas of stellar atmospheres, the Stark-broadened line profiles of resonance transitions are of the utmost importance because they give a relatively large contribution to radiative energy transport. Quite a large number of papers have been published that use different theoretical approximations to calculate these parameters, and to study their dependence on the nuclear charge number, effective ionization potential, atomic polarizability or principal quantum number [1-4]. In order to provide astrophysicists with the relevant data needed for the theoretical modelling of stellar spectra and stellar atmospheres, we made an effort to determine the relevant Stark-broadening parameters with the semi-classical formalism of $\mathrm{Pb}$ II [5]. In this work, we present Sn II semi-classical Stark-broadening calculations.
Interstellar singly-ionized tin was first detected (in several stars: 15 Mon, 23 Ori, $\pi$ Sco, 1 Sco and $\zeta$ Oph) through absorption at $1400.45 \AA$ line by Hobbs et al [6]. Savage and Sembach [7] revealed interstellar absorption lines of As II, Se II, Kr I, Sn II, T1 II and Pb II. Sofía et al [8] determined the gas-phase interstellar abundance of the Sn II in the diffuse clouds toward 14 stars. Their measures demonstrated that tin is exchanged between the gas and dust phases of the diffuse interstellar medium. Hobbs indicates that future GHRS observations of several weaker lines of Sn II as the 1757.905 \& line can be used to corroborate the identification of the $1400.45 \AA$ line. So reliable Sn II data in the UV range are needed to derive different astrophysical parameters.

Therefore, reliable Sn II Stark-broadening profiles are needed to derive various astrophysical parameters. Experimental values might be preferable to theoretical ones, but in the spectrum of the Sn II ion there are several intense lines for which no data at all can be found in the literature.

Miller et al [9] obtained experimental data with a gasdriven shock tube for ten Sn II lines between 3200 and $7900 \AA$, adjusted to an electron density of $10^{17} \mathrm{~cm}^{-3}$. The data have an estimated relative uncertainty of about $30 \%$. Lakicevic [10] 
estimated the Stark half-widths for the $1757.4 \AA$ resonant line of Sn II at $20000 \mathrm{~K}$ and $10^{17} \mathrm{~cm}^{-3}$. Puric et al [11] revised the Stark half-widths for the $6845.4 \AA$ line of Sn II using an electromagnetic shock tube. Djenize et al [12] have obtained experimental Stark broadening of several Sn II lines for a temperature of $33000 \mathrm{~K}$ in a low pressure pulsed arc. They did not provide uncertainties and their measures seem four times but small that those of other authors. Experimental Stark widths for 24 lines were presented for Martînez and Blanco [13] at $10000 \mathrm{~K}$ and $10^{16} \mathrm{~cm}^{-3}$.

In this work, we present semi-empirical Stark widths for 80 lines of Sn II arising from ns ${ }^{2} \mathbf{S}_{1 / 2}$, np ${ }^{2} \mathbf{P}_{1 / 2,3 / 2}$, nd ${ }^{2} \mathbf{D}_{3 / 2,5 / 2}$ and $n{ }^{2} \mathbf{F}_{5 / 2,7 / 2}$ levels of $\mathrm{Sn}$ II. The results have been obtained using Griem's semi-classical calculations [14]. Stark widths are presented as functions of temperature for an electron density of $10^{17} \mathrm{~cm}^{-3}$.

The theoretical values obtained in this work are compared with the experimental ones published by the authors mentioned above. The calculated values are in good agreement with the experimental data. We have studied the dependence of the Stark width $\omega(\AA)$ on principal quantum number, $n$, for several series of Sn II. Also the regularity of this parameter versus the temperature has been presented.

We also present Stark line-shifts for the 3284.3, 3352.5, $5799.0,6455.2$ and $6845.4 \AA$ lines in order to compare our method with experimental results.

\section{Theoretical calculations}

In 1968, Griem suggested a simple semi-empirical impact approximation based on Baranger's original formulation [15] and the use of an effective Gaunt factor approximation proposed by Seaton [16] and Van Regenmorter [17]. The Stark line width and Stark line-shifts can be calculated from the following semi-empirical formulae

$$
\begin{gathered}
\omega_{\mathrm{se}} \approx \\
8\left(\frac{\pi}{3}\right)^{3 / 2} \frac{h}{m a_{0}} N_{\mathrm{e}}\left(\frac{E_{H}}{k T}\right)^{1 / 2} \\
\times\left[\sum_{i^{\prime}}\left|\left\langle i^{\prime}|\vec{r}| i\right\rangle\right|^{2} g_{\mathrm{se}}\left(\frac{E}{\Delta E_{i^{\prime} i}}\right)\right. \\
\left.+\sum_{f^{\prime}}\left|\left\langle f^{\prime}|\vec{r}| f\right\rangle\right|^{2} g_{\mathrm{se}}\left(\frac{E}{\Delta E_{f^{\prime} f}}\right)\right], \\
d \approx-8\left(\frac{\pi}{3}\right)^{3 / 2} \frac{h}{m a_{0}} N_{\mathrm{e}}\left(\frac{E_{H}}{k T}\right)^{1 / 2} \\
\times\left[\sum_{i^{\prime}}\left(\frac{\Delta E_{i^{\prime} i}}{\left|\Delta E_{i^{\prime} i}\right|}\right)\left|\left\langle i^{\prime}|\vec{r}| i\right\rangle\right|^{2} g_{\mathrm{sh}}\left(\frac{E}{\Delta E_{i^{\prime} i}}\right)\right. \\
\left.-\sum_{f^{\prime}}\left(\frac{\Delta E_{f^{\prime} f}}{\left|\Delta E_{f^{\prime} f}\right|}\right)\left|\left\langle f^{\prime}|\vec{r}| f\right\rangle\right|^{2} g_{\mathrm{sh}}\left(\frac{E}{\Delta E_{f^{\prime} f}}\right)\right] .
\end{gathered}
$$

In these formulae, $\omega_{s e}$ and $d$ are the Stark line width and shifts respectively in angular frequency units, $E_{H}$ is the hydrogen ionization energy, $N_{e}$ is the free electron perturber

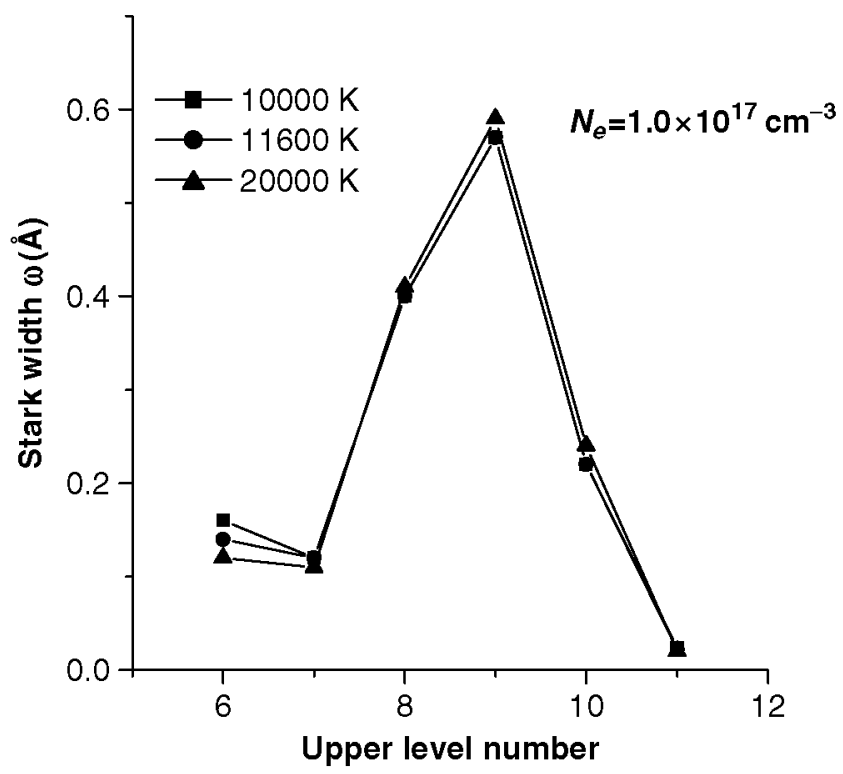

Figure 1. Stark widths FWHM $(\omega)$ for $5 p^{2} \mathrm{P}_{1 / 2}-n{ }^{2} \mathrm{~S}_{1 / 2}$ lines versus the principal quantum number of the upper state.

density, $T$ is the electron temperature, $E=(3 / 2) k T$ is the mean energy of the perturbing electron and $g_{\text {se }}$, and $g_{\text {sh }}$ are the effective Gaunt factors which are calculated and tabulated by Griem. These factors are slowly varying functions of $X_{i^{\prime} i}=E / \Delta E_{i^{\prime} i}$, where $\Delta E_{i^{\prime} i}$ is the energy difference between a perturbing level $i$ and the perturbed level $i$. The indices $i$ and $f$ denote the initial (upper) and final (lower) levels of the transitions, respectively. $\omega_{s e}$ is the half-width halfmaximum (HWHM) of the Lorentz profile in frequency units. $\omega_{s e}$ is proportional to the the full-width half-maximum (FWHM) line $\omega$ in wavelength units, through the expression $\omega=\omega_{s e} \lambda^{2} /(\pi c)$.

The atomic matrix elements were obtained using relativistic Hartree-Fock calculations and configuration interaction in an intermediate coupling (IC) scheme. The Cowan code [18] was selected for this purpose. To provide level energies for our calculations, the table of Moore [19] has been used.

The system considered is complex, with high $Z$ where both relativistic and correlation effects are expected to be important. Least-squares fitting of experimental energy levels partially account correlation effects not explicitly calculated in our work. The relatively good agreement between experimental transition probabilities and our theoretical results obtained with this method [20-23] shows that our IC mixing accounts for the correlation effects.

Singly ionized tin has a single optical electron whose energy levels are well separated. Figure 1 in [22] displays a complete scheme of the single-ionized tin energy levels. Transitions studied in this work can be seen in this figure. This structure allows us to obtain a suitable comparison between experimental and theoretical results with relativistic central field calculations using the LS coupling scheme. Nevertheless, this scheme is not sufficient to describe the $5 \mathrm{~s} 5 \mathrm{p}^{2}$. A more detailed description is necessary to take into account some features of interaction between the $5 \mathrm{~s}^{2} 5 \mathrm{~d}$ and 
Table 1. Sn II m(5-6)p ${ }^{2} \mathrm{P}_{1 / 2,3 / 2}-\mathrm{n}(6-11) \mathrm{p}{ }^{2} \mathrm{~S}_{1 / 2}$ line-widths (FWHM) normalized to $N_{e}=10^{17} \mathrm{~cm}^{-3}$.

\begin{tabular}{|c|c|c|c|c|c|}
\hline \multirow{2}{*}{$\begin{array}{l}\lambda(\AA) \\
\text { vacuum }\end{array}$} & \multicolumn{2}{|c|}{ Transition } & \multirow{2}{*}{$\begin{array}{l}\text { Temperature } \\
\left(\times 10^{3} \mathrm{~K}\right)\end{array}$} & \multicolumn{2}{|c|}{$\omega(\AA)$} \\
\hline & Upper level & Lower level & & This work & Literature \\
\hline 1757.9 & $6 s^{2} S_{1 / 2}$ & $5 \mathrm{p}^{2} \mathrm{P}_{1 / 2}$ & $\begin{array}{l}10 \\
11.6 \\
20\end{array}$ & $\begin{array}{l}0.16 \\
0.14 \\
0.12\end{array}$ & $0.11^{\mathrm{a}}$ \\
\hline 1899.9 & $6 s^{2} S_{1 / 2}$ & $5 p^{2} \mathrm{P}_{3 / 2}$ & $\begin{array}{l}10 \\
11.6 \\
20\end{array}$ & $\begin{array}{l}0.23 \\
0.22 \\
0.16\end{array}$ & \\
\hline 1159.0 & $7 \mathrm{~s}^{2} \mathrm{~S}_{1 / 2}$ & $5 p^{2} \mathrm{P}_{1 / 2}$ & $\begin{array}{l}10 \\
11.6 \\
20\end{array}$ & $\begin{array}{l}0.12 \\
0.12 \\
0.11\end{array}$ & \\
\hline 1219.1 & $7 \mathrm{~s}^{2} \mathrm{~S}_{1 / 2}$ & $5 p^{2} P_{3 / 2}$ & $\begin{array}{l}10 \\
11.6 \\
20\end{array}$ & $\begin{array}{l}0.20 \\
0.20 \\
0.17\end{array}$ & \\
\hline 6763.2 & $7 \mathrm{~s}^{2} \mathrm{~S}_{1 / 2}$ & $6 \mathrm{p}^{2} \mathrm{P}_{1 / 2}$ & $\begin{array}{l}10 \\
11.6 \\
20\end{array}$ & $\begin{array}{l}6.9 \\
6.6 \\
5.9\end{array}$ & $\begin{array}{l}5.0 \pm 0.8^{b} \\
5.5 \pm 1.6^{c}\end{array}$ \\
\hline 7192.7 & $7 \mathrm{~s}^{2} \mathrm{~S}_{1 / 2}$ & $6 \mathrm{p}^{2} \mathrm{P}_{3 / 2}$ & $\begin{array}{l}10 \\
11.6 \\
20\end{array}$ & $\begin{array}{l}9.8 \\
9.3 \\
8.1\end{array}$ & \\
\hline 1016.2 & $8 s^{2} S_{1 / 2}$ & $5 p^{2} \mathrm{P}_{1 / 2}$ & $\begin{array}{l}10 \\
11.6 \\
20\end{array}$ & $\begin{array}{l}0.40 \\
0.40 \\
0.41\end{array}$ & \\
\hline 1062.1 & $8 s^{2} S_{1 / 2}$ & $5 p^{2} \mathrm{P}_{3 / 2}$ & $\begin{array}{l}10 \\
11.6 \\
20\end{array}$ & $\begin{array}{l}0.44 \\
0.44 \\
0.50\end{array}$ & \\
\hline 3716.4 & $8 s^{2} S_{1 / 2}$ & $6 p^{2} \mathrm{P}_{1 / 2}$ & $\begin{array}{l}10 \\
11.6 \\
20\end{array}$ & $\begin{array}{l}4.6 \\
4.6 \\
4.7\end{array}$ & $3.6 \pm 0.8^{b}$ \\
\hline 3842.5 & $8 s^{2} S_{1 / 2}$ & $6 p^{2} \mathrm{P}_{3 / 2}$ & $\begin{array}{l}10 \\
11.6 \\
20\end{array}$ & $\begin{array}{l}5.6 \\
5.5 \\
5.5\end{array}$ & $3.4 \pm 0.6^{b}$ \\
\hline 955.3 & $9 \mathrm{~s}{ }^{2} S_{1 / 2}$ & $5 \mathrm{p}^{2} \mathrm{P}_{1 / 2}$ & $\begin{array}{l}10 \\
11.6 \\
20\end{array}$ & $\begin{array}{l}0.57 \\
0.57 \\
0.59\end{array}$ & \\
\hline 995.7 & $9 \mathrm{~s}^{2} \mathrm{~S}_{1 / 2}$ & $5 p^{2} P_{3 / 2}$ & $\begin{array}{l}10 \\
11.6 \\
20\end{array}$ & $\begin{array}{l}0.54 \\
0.54 \\
0.59\end{array}$ & \\
\hline 3013.3 & $9 \mathrm{~s}^{2} \mathrm{~S}_{1 / 2}$ & $6 \mathrm{p}^{2} \mathrm{P}_{1 / 2}$ & $\begin{array}{l}10 \\
11.6 \\
20\end{array}$ & $\begin{array}{l}4.9 \\
5.0 \\
5.5\end{array}$ & \\
\hline 3095.5 & $9 s^{2} S_{1 / 2}$ & $6 p^{2} \mathrm{P}_{3 / 2}$ & $\begin{array}{l}10 \\
11.6 \\
20\end{array}$ & $\begin{array}{l}5.5 \\
5.3 \\
5.8\end{array}$ & \\
\hline 922.9 & $10 \mathrm{~s}^{2} \mathrm{~S}_{1 / 2}$ & $5 p{ }^{2} P_{1 / 2}$ & $\begin{array}{l}10 \\
11.6 \\
20\end{array}$ & $\begin{array}{l}0.22 \\
0.22 \\
0.24\end{array}$ & \\
\hline 960.6 & $10 \mathrm{~s}^{2} \mathrm{~S}_{1 / 2}$ & $5 p^{2} \mathrm{P}_{3 / 2}$ & $\begin{array}{l}10 \\
11.6 \\
20\end{array}$ & $\begin{array}{l}0.24 \\
0.25 \\
0.27\end{array}$ & \\
\hline 2713.0 & $10 \mathrm{~s}^{2} \mathrm{~S}_{1 / 2}$ & $6 p^{2} \mathrm{P}_{1 / 2}$ & $\begin{array}{l}10 \\
11.6 \\
20\end{array}$ & $\begin{array}{l}2.0 \\
2.0 \\
2.2\end{array}$ & \\
\hline 2779.6 & $10 \mathrm{~s}^{2} \mathrm{~S}_{1 / 2}$ & $6 p^{2} \mathrm{P}_{3 / 2}$ & $\begin{array}{l}10 \\
11.6 \\
20\end{array}$ & $\begin{array}{l}2.2 \\
2.3 \\
2.4\end{array}$ & \\
\hline 903.4 & $11 \mathrm{~s}^{2} \mathrm{~S}_{1 / 2}$ & $5 p^{2} \mathrm{P}_{1 / 2}$ & $\begin{array}{l}10 \\
11.6 \\
20\end{array}$ & $\begin{array}{l}0.024 \\
0.022 \\
0.020\end{array}$ & \\
\hline 939.5 & $11 \mathrm{~s}^{2} \mathrm{~S}_{1 / 2}$ & $5 p^{2} P_{3 / 2}$ & $\begin{array}{l}10 \\
11.6 \\
20\end{array}$ & $\begin{array}{l}0.036 \\
0.034 \\
0.038\end{array}$ & \\
\hline 2551.0 & $11 \mathrm{~s}^{2} \mathrm{~S}_{1 / 2}$ & $6 \mathrm{p}^{2} \mathrm{P}_{1 / 2}$ & $\begin{array}{l}10 \\
11.6 \\
20\end{array}$ & $\begin{array}{l}0.35 \\
0.33 \\
0.28\end{array}$ & \\
\hline 2609.7 & $11 \mathrm{~s}^{2} \mathrm{~S}_{1 / 2}$ & $6 p^{2} \mathrm{P}_{3 / 2}$ & $\begin{array}{l}10 \\
11.6 \\
20\end{array}$ & $\begin{array}{l}0.61 \\
0.58 \\
0.48\end{array}$ & \\
\hline
\end{tabular}

${ }^{a}$ Lakicevic [10].

${ }^{b}$ Martínez and Blanco [13].

${ }^{c}$ Miller et al [9]. 
Table 2. Sn II $5 \mathrm{~s}^{2} 6 \mathrm{~s}-5 \mathrm{~s}^{2} n \mathrm{p}, 5 \mathrm{~s}^{2} 5 \mathrm{~d}-5 \mathrm{~s}^{2} n \mathrm{p}$ and $5 \mathrm{~s} 5 \mathrm{p}^{2}-5 \mathrm{~s}^{2} n \mathrm{p}$ line-widths (FWHM) normalized to $N_{e}=10^{17} \mathrm{~cm}^{-3}$.

\begin{tabular}{|c|c|c|c|c|c|}
\hline \multirow{2}{*}{$\begin{array}{l}\lambda(\AA) \\
\text { vacuum }\end{array}$} & \multicolumn{2}{|c|}{ Transition } & \multirow{2}{*}{$\begin{array}{l}\text { Temperature } \\
\left(\times 10^{3} \mathrm{~K}\right)\end{array}$} & \multicolumn{2}{|c|}{$\omega(\AA)$} \\
\hline & Upper level & Lower level & & This work & Literature \\
\hline \multirow[t]{6}{*}{6845.38} & $6 p^{2} P_{1 / 2}$ & $6 s^{2} S_{1 / 2}$ & 10 & 3.8 & $4.0 \pm 0.6^{\mathrm{a}}$ \\
\hline & & & 11.6 & 3.5 & $4.2 \pm 1.6^{\mathrm{b}}$ \\
\hline & & & 16 & 3.1 & $3.3 \pm 0.5^{c}$ \\
\hline & & & 20 & 2.8 & $2.3 \pm 0.3^{c}$ \\
\hline & & & 26 & 2.5 & \\
\hline & & & 33 & 2.4 & $0.652^{\mathrm{d}}$ \\
\hline \multirow[t]{6}{*}{6455.2} & $6 \mathrm{p}^{2} \mathrm{P}_{3 / 2}$ & $6 s^{2} S_{1 / 2}$ & 10 & 4.7 & $2.8 \pm 0.6^{\mathrm{a}}$ \\
\hline & & & 11.6 & 4.4 & \\
\hline & & & 16 & 3.8 & \\
\hline & & & 20 & 3.3 & \\
\hline & & & 26 & 3.2 & \\
\hline & & & 33 & 3.0 & $0.496^{\mathrm{d}}$ \\
\hline \multirow[t]{6}{*}{4619.5} & $6 p^{2} \mathrm{P}_{3 / 2}$ & $5 p^{2}{ }^{4} P_{5 / 2}$ & 10 & 2.7 & \\
\hline & & & 11.6 & 2.5 & $1.6 \pm 0.5^{b}$ \\
\hline & & & 16 & 1.1 & \\
\hline & & & 20 & 1.9 & \\
\hline & & & 26 & 1.7 & \\
\hline & & & 33 & 1.6 & \\
\hline \multirow[t]{6}{*}{2855.7} & $7 \mathrm{p}^{2} \mathrm{P}_{1 / 2}$ & $6 s{ }^{2} S_{1 / 2}$ & 10 & 1.6 & \\
\hline & & & 11.6 & 1.5 & \\
\hline & & & 16 & 1.5 & \\
\hline & & & 20 & 1.5 & \\
\hline & & & 26 & 1.5 & \\
\hline & & & 33 & 1.5 & \\
\hline \multirow[t]{6}{*}{4878.7} & $7 \mathrm{p}^{2} \mathrm{P}_{1 / 2}$ & $5 \mathrm{~d}^{2} \mathrm{D}_{3 / 2}$ & 10 & 5.1 & $4.4 \pm 0.6^{\mathrm{a}}$ \\
\hline & & & 11.6 & 5.0 & \\
\hline & & & 16 & 4.9 & \\
\hline & & & 20 & 4.9 & \\
\hline & & & 26 & 4.8 & \\
\hline & & & 33 & 4.8 & \\
\hline \multirow[t]{6}{*}{3024.8} & $7 \mathrm{p}^{2} \mathrm{P}_{1 / 2}$ & $5 p^{2}{ }^{2} D_{3 / 2}$ & 10 & 1,8 & \\
\hline & & & 11.6 & 1,8 & \\
\hline & & & 16 & 1,7 & \\
\hline & & & 20 & 1,7 & \\
\hline & & & 26 & 1,7 & \\
\hline & & & 33 & 1,7 & \\
\hline \multirow[t]{6}{*}{2826.4} & $7 p^{2} \mathrm{P}_{3 / 2}$ & $6 s{ }^{2} S_{1 / 2}$ & 10 & 2.8 & \\
\hline & & & 11.6 & 2.8 & \\
\hline & & & 16 & 2.8 & \\
\hline & & & 20 & 2.7 & \\
\hline & & & 26 & 2.7 & \\
\hline & & & 33 & 2.7 & \\
\hline \multirow[t]{6}{*}{4793.8} & $7 p^{2} \mathrm{P}_{3 / 2}$ & $5 \mathrm{~d}^{2} \mathrm{D}_{3 / 2}$ & 10 & 8.4 & \\
\hline & & & 11.6 & 7.9 & \\
\hline & & & 16 & 6.7 & \\
\hline & & & 20 & 6.0 & \\
\hline & & & 26 & 5.3 & \\
\hline & & & 33 & 4.7 & \\
\hline \multirow[t]{6}{*}{4946.0} & $7 p^{2} \mathrm{P}_{3 / 2}$ & $5 d^{2} D_{5 / 2}$ & 10 & 9.7 & $4.0 \pm 0.8^{\mathrm{a}}$ \\
\hline & & & 11.6 & 9.1 & \\
\hline & & & 16 & 7.7 & \\
\hline & & & 20 & 6.9 & \\
\hline & & & 26 & 6.0 & \\
\hline & & & 33 & 5.4 & \\
\hline \multirow[t]{6}{*}{2992.0} & $7 p^{2} \mathrm{P}_{3 / 2}$ & $5 p^{2}{ }^{2} D_{3 / 2}$ & 10 & 3.2 & \\
\hline & & & 11.6 & 3.1 & \\
\hline & & & 16 & 3.1 & \\
\hline & & & 20 & 3.1 & \\
\hline & & & 26 & 3.1 & \\
\hline & & & 33 & 3.1 & \\
\hline
\end{tabular}


Table 2. Continued.

\begin{tabular}{|c|c|c|c|c|c|}
\hline \multirow{2}{*}{$\begin{array}{l}\lambda(\AA) \\
\text { vacuum }\end{array}$} & \multicolumn{2}{|c|}{ Transition } & \multirow{2}{*}{$\begin{array}{l}\text { Temperature } \\
\left(\times 10^{3} \mathrm{~K}\right)\end{array}$} & \multicolumn{2}{|c|}{$\omega(\AA)$} \\
\hline & Upper level & Lower level & & This work & Literature \\
\hline \multirow[t]{6}{*}{3048.5} & $7 p^{2} \mathrm{P}_{3 / 2}$ & $5 p^{2}{ }^{2} D_{5 / 2}$ & 10 & 3.5 & \\
\hline & & & 11.6 & 3.4 & \\
\hline & & & 16 & 3.3 & \\
\hline & & & 20 & 3.3 & \\
\hline & & & 26 & 3.3 & \\
\hline & & & 33 & 3.4 & \\
\hline \multirow[t]{6}{*}{2256.8} & $8 p^{2} P_{1 / 2}$ & $6 s^{2} S_{1 / 2}$ & 10 & 3.1 & \\
\hline & & & 11.6 & 3.1 & \\
\hline & & & 16 & 3.1 & \\
\hline & & & 20 & 3.1 & \\
\hline & & & 26 & 3.1 & \\
\hline & & & 33 & 3.1 & \\
\hline \multirow[t]{6}{*}{3356.8} & $8 p^{2} \mathrm{P}_{1 / 2}$ & $5 d^{2} D_{3 / 2}$ & 10 & 7.0 & \\
\hline & & & 11.6 & 7.0 & \\
\hline & & & 16 & 7.1 & \\
\hline & & & 20 & 7.1 & \\
\hline & & & 26 & 7.0 & \\
\hline & & & 33 & 6.7 & \\
\hline \multirow[t]{6}{*}{2361.1} & $8 p^{2} P_{1 / 2}$ & $5 p^{2}{ }^{2} D_{3 / 2}$ & 10 & 3.4 & \\
\hline & & & 11.6 & 3.4 & \\
\hline & & & 16 & 3.5 & \\
\hline & & & 20 & 3.5 & \\
\hline & & & 26 & 3.4 & \\
\hline & & & 33 & 3.3 & \\
\hline \multirow[t]{6}{*}{2247.1} & $8 p^{2} P_{3 / 2}$ & $6 s^{2} S_{1 / 2}$ & 10 & 5.6 & \\
\hline & & & 11.6 & 5.7 & \\
\hline & & & 16 & 5.8 & \\
\hline & & & 20 & 5.9 & \\
\hline & & & 26 & 5.9 & \\
\hline & & & 33 & 5.8 & \\
\hline \multirow[t]{6}{*}{3335.4} & $8 p^{2} \mathrm{P}_{3 / 2}$ & $5 d^{2} D_{3 / 2}$ & 10 & 9.4 & \\
\hline & & & 11.6 & 9.5 & \\
\hline & & & 16 & 9.7 & \\
\hline & & & 20 & 9.8 & \\
\hline & & & 26 & 9.8 & \\
\hline & & & 33 & 9.7 & \\
\hline \multirow[t]{6}{*}{3408.4} & $8 p^{2} \mathrm{P}_{3 / 2}$ & $5 d^{2} D_{5 / 2}$ & 10 & 10.0 & \\
\hline & & & 11.6 & 10.1 & \\
\hline & & & 16 & 10.3 & \\
\hline & & & 20 & 10.4 & \\
\hline & & & 26 & 10.4 & \\
\hline & & & 33 & 10.2 & \\
\hline \multirow[t]{6}{*}{2350.6} & $8 p^{2} \mathrm{P}_{3 / 2}$ & $5 p^{2}{ }^{2} D_{3 / 2}$ & 10 & 4.6 & \\
\hline & & & 11.6 & 4.7 & \\
\hline & & & 16 & 4.8 & \\
\hline & & & 20 & 4.8 & \\
\hline & & & 26 & 4.8 & \\
\hline & & & 33 & 4.8 & \\
\hline \multirow[t]{6}{*}{2385.3} & $8 p^{2} \mathrm{P}_{3 / 2}$ & $5 p^{2}{ }^{2} D_{5 / 2}$ & 10 & 4.8 & \\
\hline & & & 11.6 & 4.9 & \\
\hline & & & 16 & 5.0 & \\
\hline & & & 20 & 5.0 & \\
\hline & & & 26 & 5.0 & \\
\hline & & & 33 & 5.0 & \\
\hline
\end{tabular}

${ }^{a}$ Martínez and Blanco [13].

${ }^{\mathrm{b}}$ Miller et al [9].

'Puric et al [11].

-Djenize et al [12]. 
Table 3. Sn II $m(5-6) \mathrm{p}{ }^{2} \mathrm{P}_{1 / 2,3 / 2}-n(5-10) \mathrm{d}^{2} \mathrm{D}_{3 / 2,5 / 2}$ line-widths (FWHM) normalized to $N_{e}=10^{17} \mathrm{~cm}^{-3}$.

\begin{tabular}{|c|c|c|c|c|c|}
\hline \multirow{2}{*}{$\begin{array}{l}\lambda(\AA) \\
\text { Vacuum }\end{array}$} & \multicolumn{2}{|c|}{ Transition } & \multirow{2}{*}{$\begin{array}{l}\text { Temperature } \\
\left(\times 10^{3} \mathrm{~K}\right)\end{array}$} & \multicolumn{2}{|c|}{$\omega(\AA)$} \\
\hline & Upper level & Lower level & & This work & Literature \\
\hline \multirow[t]{3}{*}{1400.4} & $5 \mathrm{~d}^{2} \mathrm{D}_{3 / 2}$ & $5 p^{2} P_{1 / 2}$ & 10 & 0.14 & \\
\hline & & & 11.6 & 0.13 & \\
\hline & & & 20 & 0.10 & \\
\hline \multirow[t]{3}{*}{1489.1} & $5 d^{2} D_{3 / 2}$ & $5 p^{2} \mathrm{P}_{3 / 2}$ & 10 & 0.18 & \\
\hline & & & 11.6 & 0.17 & \\
\hline & & & 20 & 0.13 & \\
\hline \multirow[t]{3}{*}{1475.0} & $5 \mathrm{~d}^{2} \mathrm{D}_{5 / 2}$ & $5 p^{2} P_{3 / 2}$ & 10 & 0.30 & \\
\hline & & & 11.6 & 0.22 & \\
\hline & & & 20 & 0.17 & \\
\hline \multirow{3}{*}{1108.2} & $6 \mathrm{~d}^{2} \mathrm{D}_{3 / 2}$ & $5 p^{2} P_{1 / 2}$ & 10 & 0.29 & \\
\hline & & & 11.6 & 0.29 & \\
\hline & & & 20 & 0.29 & \\
\hline \multirow[t]{3}{*}{1162.9} & $6 d^{2} D_{3 / 2}$ & $5 p^{2} P_{3 / 2}$ & 10 & 0.34 & \\
\hline & & & 11.6 & 0.32 & \\
\hline & & & 20 & 0.32 & \\
\hline \multirow[t]{3}{*}{5333.9} & $6 \mathrm{~d}^{2} \mathrm{D}_{3 / 2}$ & $6 p^{2} \mathrm{P}_{1 / 2}$ & 10 & 7.2 & $6.0 \pm 0.8^{\mathrm{a}}$ \\
\hline & & & 11.6 & 7.2 & $5.3 \pm 0.7^{b}$ \\
\hline & & & 20 & 7.1 & \\
\hline \multirow[t]{3}{*}{5597,6} & $6 \mathrm{~d}^{2} \mathrm{D}_{3 / 2}$ & $6 p^{2} \mathrm{P}_{3 / 2}$ & 10 & $7.4^{*}$ & $4.6 \pm 0.6^{\mathrm{a}}$ \\
\hline & & & 11.6 & $7.3^{*}$ & \\
\hline & & & 20 & $7.1^{*}$ & \\
\hline \multirow[t]{3}{*}{1161.4} & $6 \mathrm{~d}^{2} \mathrm{D}_{5 / 2}$ & $5 p^{2} P_{3 / 2}$ & 10 & 0.45 & \\
\hline & & & 11.6 & 0.45 & \\
\hline & & & 20 & 0.45 & \\
\hline \multirow[t]{3}{*}{5562.9} & $6 \mathrm{~d}^{2} \mathrm{D}_{5 / 2}$ & $6 p^{2} \mathrm{P}_{3 / 2}$ & 10 & $7.3^{*}$ & $5.2 \pm 0.8^{\mathrm{a}}$ \\
\hline & & & 11.6 & $6.8^{*}$ & $5.1 \pm 0.7^{\mathrm{b}}$ \\
\hline & & & 20 & $5.6^{*}$ & \\
\hline \multirow[t]{3}{*}{997.2} & $7 \mathrm{~d}^{2} \mathrm{D}_{3 / 2}$ & $5 p^{2} \mathrm{P}_{1 / 2}$ & 10 & $0.28^{*}$ & \\
\hline & & & 11.6 & $0.28^{*}$ & \\
\hline & & & 20 & $0.26^{*}$ & \\
\hline 1041.3 & $7 \mathrm{~d}^{2} \mathrm{D}_{3 / 2}$ & $5 p^{2} P_{3 / 2}$ & 10 & $0.31^{*}$ & \\
\hline & & & 11.6 & $0.30^{*}$ & \\
\hline & & & 20 & $0.28 *$ & \\
\hline 3473.4 & $7 \mathrm{~d}^{2} \mathrm{D}_{3 / 2}$ & $6 p^{2} \mathrm{P}_{1 / 2}$ & 10 & $3.8^{*}$ & $4.2 \pm 0.8^{\mathrm{a}}$ \\
\hline & & & 11.6 & $3.8^{*}$ & \\
\hline & & & 20 & $3.5^{*}$ & \\
\hline 3583.3 & $7 \mathrm{~d}^{2} \mathrm{D}_{3 / 2}$ & $6 p^{2} \mathrm{P}_{3 / 2}$ & 10 & $4.0^{*}$ & $3.6 \pm 0.8^{\mathrm{a}}$ \\
\hline & & & 11.6 & $4.1^{*}$ & \\
\hline & & & 20 & $3.8^{*}$ & \\
\hline 1040.7 & $7 \mathrm{~d}^{2} \mathrm{D}_{5 / 2}$ & $5 p^{2} \mathrm{P}_{3 / 2}$ & 10 & $0.29^{*}$ & \\
\hline & & & 11.6 & $0.29^{*}$ & \\
\hline & & & 20 & $0.27^{*}$ & \\
\hline 3576.3 & $7 \mathrm{~d}^{2} \mathrm{D}_{5 / 2}$ & $6 p^{2} P_{3 / 2}$ & 10 & $3.9^{*}$ & $4.6 \pm 0.6^{\mathrm{a}}$ \\
\hline & & & 11.6 & $3.9^{*}$ & $3.0 \pm 1.0^{\mathrm{b}}$ \\
\hline & & & 20 & $3.8^{*}$ & \\
\hline 945.8 & $8 \mathrm{~d}^{2} \mathrm{D}_{3 / 2}$ & $5 p^{2} P_{1 / 2}$ & 10 & $0.68^{*}$ & \\
\hline & & & 11.6 & $0.65^{*}$ & \\
\hline & & & 20 & $0.50^{*}$ & \\
\hline 985.4 & $8 \mathrm{~d}^{2} \mathrm{D}_{3 / 2}$ & $5 p^{2} P_{3 / 2}$ & 10 & $0.74^{*}$ & \\
\hline & & & 11.6 & $0.71^{*}$ & \\
\hline & & & 20 & $0.55^{*}$ & \\
\hline 2920.7 & $8 \mathrm{~d}^{2} \mathrm{D}_{3 / 2}$ & $6 p^{2} \mathrm{P}_{1 / 2}$ & 10 & $6.9^{*}$ & \\
\hline & & & 11.6 & $6.6^{*}$ & \\
\hline & & & 20 & $5.1^{*}$ & \\
\hline 2998.0 & $8 \mathrm{~d}^{2} \mathrm{D}_{3 / 2}$ & $6 p^{2} \mathrm{P}_{3 / 2}$ & 10 & $7.2^{*}$ & \\
\hline & & & 11.6 & $6.9^{*}$ & \\
\hline & & & 20 & $5.4^{*}$ & \\
\hline 985.1 & $8 \mathrm{~d}^{2} \mathrm{D}_{5 / 2}$ & $5 p^{2} P_{3 / 2}$ & 10 & $0.72^{*}$ & \\
\hline & & & 11.6 & $0.69^{*}$ & \\
\hline & & & 20 & $0.55^{*}$ & \\
\hline 2995.0 & $8 d^{2} D_{5 / 2}$ & $6 p^{2} P_{3 / 2}$ & 10 & $7.0^{*}$ & $8.6 \pm 1.2^{\mathrm{a}}$ \\
\hline & & & 11.6 & $6.8^{*}$ & \\
\hline & & & 20 & $5.4^{*}$ & \\
\hline
\end{tabular}


Table 3. Continued.

\begin{tabular}{|c|c|c|c|c|c|}
\hline \multirow{2}{*}{$\begin{array}{l}\lambda(\AA) \\
\text { Vacuum }\end{array}$} & \multicolumn{2}{|c|}{ Transition } & \multirow{2}{*}{$\begin{array}{l}\text { Temperature } \\
\left(\times 10^{3} \mathrm{~K}\right)\end{array}$} & \multicolumn{2}{|c|}{$\omega(\AA)$} \\
\hline & Upper level & Lower level & & This work & Literature \\
\hline 917.4 & $9 \mathrm{~d}^{2} \mathrm{D}_{3 / 2}$ & $5 p^{2} \mathrm{P}_{1 / 2}$ & $\begin{array}{l}10 \\
11.6 \\
20\end{array}$ & $\begin{array}{l}0.24 \\
0.24 \\
0.26\end{array}$ & \\
\hline 954.6 & $9 d^{2} D_{5 / 2}$ & $5 \mathrm{p}^{2} \mathrm{P}_{3 / 2}$ & $\begin{array}{l}10 \\
11.6 \\
20\end{array}$ & $\begin{array}{l}0.28 \\
0.28 \\
0.28\end{array}$ & \\
\hline 954.4 & $9 \mathrm{~d}^{2} \mathrm{D}_{5 / 2}$ & $5 \mathrm{p}^{2} \mathrm{P}_{3 / 2}$ & $\begin{array}{l}10 \\
11.6 \\
20\end{array}$ & $\begin{array}{l}0.40 \\
0.40 \\
0.42\end{array}$ & \\
\hline 899.9 & $10 \mathrm{~d}^{2} \mathrm{D}_{3 / 2}$ & $5 p^{2} \mathrm{P}_{1 / 2}$ & $\begin{array}{l}10 \\
11.6 \\
20\end{array}$ & $\begin{array}{l}0.028 \\
0.026 \\
0.024\end{array}$ & \\
\hline 935.7 & $10 \mathrm{~d}^{2} \mathrm{D}_{3 / 2}$ & $5 p^{2} \mathrm{P}_{3 / 2}$ & $\begin{array}{l}10 \\
11.6 \\
20\end{array}$ & $\begin{array}{l}0.042 \\
0.038 \\
0.034\end{array}$ & \\
\hline 935.5 & $10 \mathrm{~d}^{2} \mathrm{D}_{5 / 2}$ & $5 p^{2} \mathrm{P}_{3 / 2}$ & $\begin{array}{l}10 \\
11.6 \\
20\end{array}$ & $\begin{array}{l}0.050 \\
0.048 \\
0.042\end{array}$ & \\
\hline
\end{tabular}

${ }^{a}$ Martínez and Blanco [13].

${ }^{\mathrm{b}}$ Miller et al $[9]$.

Table 4. Sn II $5 \mathrm{~s} 5 \mathrm{p}^{22} \mathrm{D}_{3 / 2,5 / 2}, 5 \mathrm{~s}^{2} 5 \mathrm{~d}^{2} \mathrm{D}_{3 / 2,5 / 2}-5 \mathrm{~s}^{2} n \mathrm{f}(4-5)^{2} \mathrm{~F}_{5 / 2,7 / 2}$ line-widths (FWHM) normalized to $N_{e}=10^{17} \mathrm{~cm}^{-3}$.

\begin{tabular}{|c|c|c|c|c|c|}
\hline \multirow{2}{*}{$\begin{array}{l}\lambda(\AA) \\
\text { vacuum }\end{array}$} & \multicolumn{2}{|c|}{ Transition } & \multirow{2}{*}{$\begin{array}{l}\text { Temperature } \\
\left(\times 10^{3} \mathrm{~K}\right)\end{array}$} & \multicolumn{2}{|c|}{$\omega(\AA)$} \\
\hline & Upper level & Lower level & & This work & Literature \\
\hline \multirow[t]{6}{*}{3284.3} & $4 \mathrm{f}^{2} \mathrm{~F}_{5 / 2}$ & $5 p^{2}{ }^{2} D_{3 / 2}$ & 10 & 1.6 & \\
\hline & & & 11.6 & 1.5 & $2.3 \pm 0.8^{\mathrm{a}}$ \\
\hline & & & 16 & 1.5 & \\
\hline & & & 20 & 1.4 & \\
\hline & & & 26 & 1.3 & \\
\hline & & & 33 & 1.2 & $0.21^{\mathrm{b}}$ \\
\hline \multirow[t]{6}{*}{3352.5} & $4 \mathrm{f}^{2} \mathrm{~F}_{5 / 2}$ & $5 p^{2}{ }^{2} D_{5 / 2}$ & 10 & 2.4 & \\
\hline & & & 11.6 & 2.4 & $2.5 \pm 0.8^{\mathrm{a}}$ \\
\hline & & & 16 & 2.3 & \\
\hline & & & 20 & 2.2 & \\
\hline & & & 26 & 2.1 & \\
\hline & & & 33 & 1.9 & $0.25^{b}$ \\
\hline \multirow[t]{6}{*}{5590.8} & $4 f^{2} F_{5 / 2}$ & $5 \mathrm{~d}^{2} \mathrm{D}_{3 / 2}$ & 10 & 4.7 & $3.8 \pm 0.6^{\mathrm{c}}$ \\
\hline & & & 11.6 & 4.6 & $3.8 \pm 1.0^{\mathrm{a}}$ \\
\hline & & & 16 & 4.4 & \\
\hline & & & 20 & 4.2 & \\
\hline & & & 26 & 3.9 & \\
\hline & & & 33 & 3.6 & $0.77^{\mathrm{b}}$ \\
\hline \multirow[t]{6}{*}{5799.0} & $4 \mathrm{f}^{2} \mathrm{~F}_{5 / 2}$ & $5 d^{2} D_{5 / 2}$ & 10 & $4.8^{*}$ & $3.6 \pm 0.6^{\mathrm{c}}$ \\
\hline & & & 11.6 & $4.6^{*}$ & \\
\hline & & & 16 & $4.2^{*}$ & \\
\hline & & & 20 & $4.1^{*}$ & \\
\hline & & & 26 & $3.8^{*}$ & \\
\hline & & & 33 & $3.3^{*}$ & $0.66^{\mathrm{b}}$ \\
\hline \multirow[t]{6}{*}{3353.2} & $4 \mathrm{f}^{2} \mathrm{~F}_{7 / 2}$ & $5 p^{2}{ }^{2} D_{5 / 2}$ & 10 & 2.0 & \\
\hline & & & 11.6 & 2.0 & \\
\hline & & & 16 & 1.9 & \\
\hline & & & 20 & 1.9 & \\
\hline & & & 26 & 1.8 & \\
\hline & & & 33 & 1.7 & \\
\hline \multirow[t]{6}{*}{5801.0} & $4 \mathrm{f}^{2} \mathrm{~F}_{7 / 2}$ & $5 \mathrm{~d}^{2} \mathrm{D}_{5 / 2}$ & 10 & $5.2^{*}$ & $3.4 \pm 0.8^{c}$ \\
\hline & & & 11.6 & $5.0^{*}$ & $4.2 \pm 1.2^{\mathrm{a}}$ \\
\hline & & & 16 & $4.8^{*}$ & \\
\hline & & & 20 & $4.6^{*}$ & \\
\hline & & & 26 & $4.4^{*}$ & \\
\hline & & & 33 & $4.1^{*}$ & \\
\hline
\end{tabular}


Table 4. Continued.

\begin{tabular}{|c|c|c|c|c|c|}
\hline \multirow{2}{*}{$\begin{array}{l}\lambda(\AA) \\
\text { vacuum }\end{array}$} & \multicolumn{2}{|c|}{ Transition } & \multirow{2}{*}{$\begin{array}{l}\text { Temperature } \\
\left(\times 10^{3} \mathrm{~K}\right)\end{array}$} & \multicolumn{2}{|c|}{$\omega(\AA)$} \\
\hline & Upper level & Lower level & & This work & Literature \\
\hline \multirow[t]{6}{*}{2449.8} & $5 f^{2} F_{5 / 2}$ & $5 p^{2}{ }^{2} D_{3 / 2}$ & 10 & $1.4^{*}$ & \\
\hline & & & 11.6 & $1.4^{*}$ & \\
\hline & & & 16 & $1.3^{*}$ & \\
\hline & & & 20 & $1.3^{*}$ & \\
\hline & & & 26 & $1.1^{*}$ & \\
\hline & & & 33 & $1.0^{*}$ & \\
\hline \multirow[t]{6}{*}{2487.6} & $5 f^{2} F_{5 / 2}$ & $5 p^{2}{ }^{2} D_{5 / 2}$ & 10 & $1.4^{*}$ & \\
\hline & & & 11.6 & $1.4^{*}$ & \\
\hline & & & 16 & $1.4^{*}$ & \\
\hline & & & 20 & $1.3^{*}$ & \\
\hline & & & 26 & $1.2^{*}$ & \\
\hline & & & 33 & $1.0^{*}$ & \\
\hline \multirow[t]{6}{*}{3538.9} & $5 f^{2} F_{5 / 2}$ & $5 d^{2} D_{3 / 2}$ & 10 & $2.9^{*}$ & $3.6 \pm 0.6^{\mathrm{c}}$ \\
\hline & & & 11.6 & $2.9^{*}$ & \\
\hline & & & 16 & $2.8^{*}$ & \\
\hline & & & 20 & $2.7^{*}$ & \\
\hline & & & 26 & $2.5^{*}$ & \\
\hline & & & 33 & $2.1^{*}$ & \\
\hline \multirow[t]{6}{*}{3621.2} & $5 f^{2} F_{5 / 2}$ & $5 d^{2} D_{5 / 2}$ & 10 & $3.0^{*}$ & \\
\hline & & & 11.6 & $3.0^{*}$ & \\
\hline & & & 16 & $3.0^{*}$ & \\
\hline & & & 20 & $2.8^{*}$ & \\
\hline & & & 26 & $2.5^{*}$ & \\
\hline & & & 33 & $2.2^{*}$ & \\
\hline \multirow[t]{6}{*}{2487.8} & $5 \mathrm{f}^{2} \mathrm{~F}_{7 / 2}$ & $5 p^{2}{ }^{2} D_{5 / 2}$ & 10 & $1.3^{*}$ & \\
\hline & & & 11.6 & $1.4^{*}$ & \\
\hline & & & 16 & $1.3^{*}$ & \\
\hline & & & 20 & $1.3^{*}$ & \\
\hline & & & 26 & $1.2^{*}$ & \\
\hline & & & 33 & $1.0^{*}$ & \\
\hline \multirow[t]{6}{*}{3621.7} & $5 \mathrm{f}^{2} \mathrm{~F}_{7 / 2}$ & $5 d^{2} D_{5 / 2}$ & 10 & $2.9^{*}$ & $3.8 \pm 0.8^{c}$ \\
\hline & & & 11.6 & $2.9^{*}$ & \\
\hline & & & 16 & $2.9^{*}$ & \\
\hline & & & 20 & $2.7^{*}$ & \\
\hline & & & 26 & $2.5^{*}$ & \\
\hline & & & 33 & $2.2^{*}$ & \\
\hline
\end{tabular}

${ }^{a}$ Miller et al [9].

${ }^{\mathrm{b}}$ Djenize et al [12].

'Martínez and Blanco [13].

Table 5. The Stark shifts normalized to $N_{e}=10^{17} \mathrm{~cm}^{-3}$.

\begin{tabular}{|c|c|c|c|c|c|}
\hline \multirow{2}{*}{$\begin{array}{l}\lambda(\AA) \\
\text { vacuum }\end{array}$} & \multicolumn{2}{|c|}{ Transition } & \multirow{2}{*}{$\begin{array}{l}\text { Temperature } \\
\left(\times 10^{3} \mathrm{~K}\right)\end{array}$} & \multicolumn{2}{|c|}{$d(\AA)$} \\
\hline & Upper level & Lower level & & This work & Literature \\
\hline \multirow[t]{6}{*}{6845.4} & $6 p^{2} \mathrm{P}_{1 / 2}$ & $6 p^{2} S_{1 / 2}$ & 10 & 0.65 & \\
\hline & & & 11.6 & 0.62 & \\
\hline & & & 16 & 0.52 & $0.90 \pm 0.13^{\mathrm{a}}$ \\
\hline & & & 20 & 0.49 & $0.76 \pm 0.11^{\mathrm{a}}$ \\
\hline & & & 26 & 0.44 & \\
\hline & & & 33 & 0.42 & $0.18^{\mathrm{b}}$ \\
\hline 6455.2 & $6 \mathrm{p}^{2} \mathrm{P}_{3 / 2}$ & $6 p^{2} S_{1 / 2}$ & 33 & 0.27 & $0.13^{\mathrm{b}}$ \\
\hline 3284.3 & $4 \mathrm{f}^{2} \mathrm{~F}_{5 / 2}$ & $5 p^{2}{ }^{2} D_{3 / 2}$ & 33 & -0.45 & $-0.05^{\mathrm{b}}$ \\
\hline 3352.5 & $4 \mathrm{f}^{2} \mathrm{~F}_{5 / 2}$ & $5 p^{2}{ }^{2} D_{5 / 2}$ & 33 & -0.40 & $-0.05^{\mathrm{b}}$ \\
\hline 5799.0 & $4 f^{2} F_{5 / 2}$ & $5 p^{2}{ }^{2} D_{5 / 2}$ & 33 & -1.12 & $-0.16^{\mathrm{b}}$ \\
\hline
\end{tabular}

Note: a positive Stark shift is blue.

${ }^{a}$ Lakicevic and Puric [25].

${ }^{\mathrm{b}}$ Djenize et al [12]. 
$5 s 5 p^{2}$ configurations and some experimental results such as the existence of intense dipole-forbidden transitions.

In this way we obtained the LS composition of each level and the degree of configuration mixing, when we consider their interaction. For the HFR calculations the Cowan computer code provides us with the radial parts of the wavefunctions for determination of transition probabilities and initial estimated of the parameters for the IC fittings. Colón and Alonso-Medina [22, 23] presented the most important features obtained from our calculations about the configuration mixing of Sn II levels.

\section{Results and discussion}

Our results for the Stark line width at a perturber density of $10^{17} \mathrm{~cm}^{-3}$ and several temperatures $T=10000-33000 \mathrm{~K}$ are displayed in column five of tables 1-4. Columns 1, 2 and 3 give the wavelengths, and the corresponding upper and lower levels respectively. Temperatures are shown in column 4 . The remaining sixth column contains the scarce experimental values to be found in the literature together with their estimated relative uncertainties. We have not included in the tables the theoretical values existing in the literature. These theoretical values in the bibliography correspond to measured Stark broadening and they are in good agreement with our results.

In table 1 , we present results for transitions arising from $n s{ }^{2} \mathbf{S}_{1 / 2} \quad(n=6-11)$. Column 4 is used for temperature, values while columns 5 and 6 are used for our results and bibliographical values respectively. One can conclude that our values are in agreement, within the margins of the Griem's semi-classical calculations [14], with the existing experimental data found. In figure 1, Stark regularity of Stark widths FWHM $(\omega)$ for $5 p{ }^{2} \mathrm{P}_{1 / 2}-n s{ }^{2} \mathrm{~S}_{1 / 2}$ lines versus the principal quantum number of the upper state, are presented. A compensation between the temperature dependence in the $\left(E_{H} / K T\right)^{1 / 2}$ factor and the behaviour of the Gaunt factors explains that the three curves presented nearly coincide for $n=6,7,8,9$. There are no experimental data to compare with our theoretical values. As can be observed, clear trends in the Stark widths are seen in our results.

In table 2, we present results for transitions arising from $n \mathrm{p}{ }^{2} \mathrm{P}_{1 / 2,3 / 2}(n=6-8)$ to $6 \mathrm{~s}^{2} \mathrm{~S}_{1 / 2}, 5 \mathrm{p}^{2}{ }^{4} \mathrm{P}_{5 / 2}, 5 \mathrm{~d}^{2} \mathrm{D}_{3 / 2,5 / 2}$ and $5 \mathrm{p}^{22} \mathrm{D}_{3 / 2,5 / 2}$. In the columns 5 and 6 , values are presented for the six temperatures with available experimental results in the literature. Except for the value obtained for $4946.0 \AA$ line a factor 2 larger to the experimental value found in the literature, the rest of the values are in agreement with the measured values. This discrepancy can be explained from our experimental level fitting method. As was indicated in our recent work [22], the $5 \mathrm{~s}^{2} 5 \mathrm{~d}^{2} \mathrm{D}_{3 / 2}$ are very mixed with the $5 \mathrm{~s}$ $5 p^{2}{ }^{2} D_{3 / 2}$ and as is well known a relatively small discrepancy between experimental and calculated energy levels can induce a relatively large discrepancy between experimental and calculated matrix elements used to calculate Stark width. Nevertheless for 6845.38 and $6455.2 \AA$ lines, whose has been measured to different temperatures for different authors $[9,11-13,24]$, our results are in very good agreement with the experimental data (data presented for Djenize et al [12]
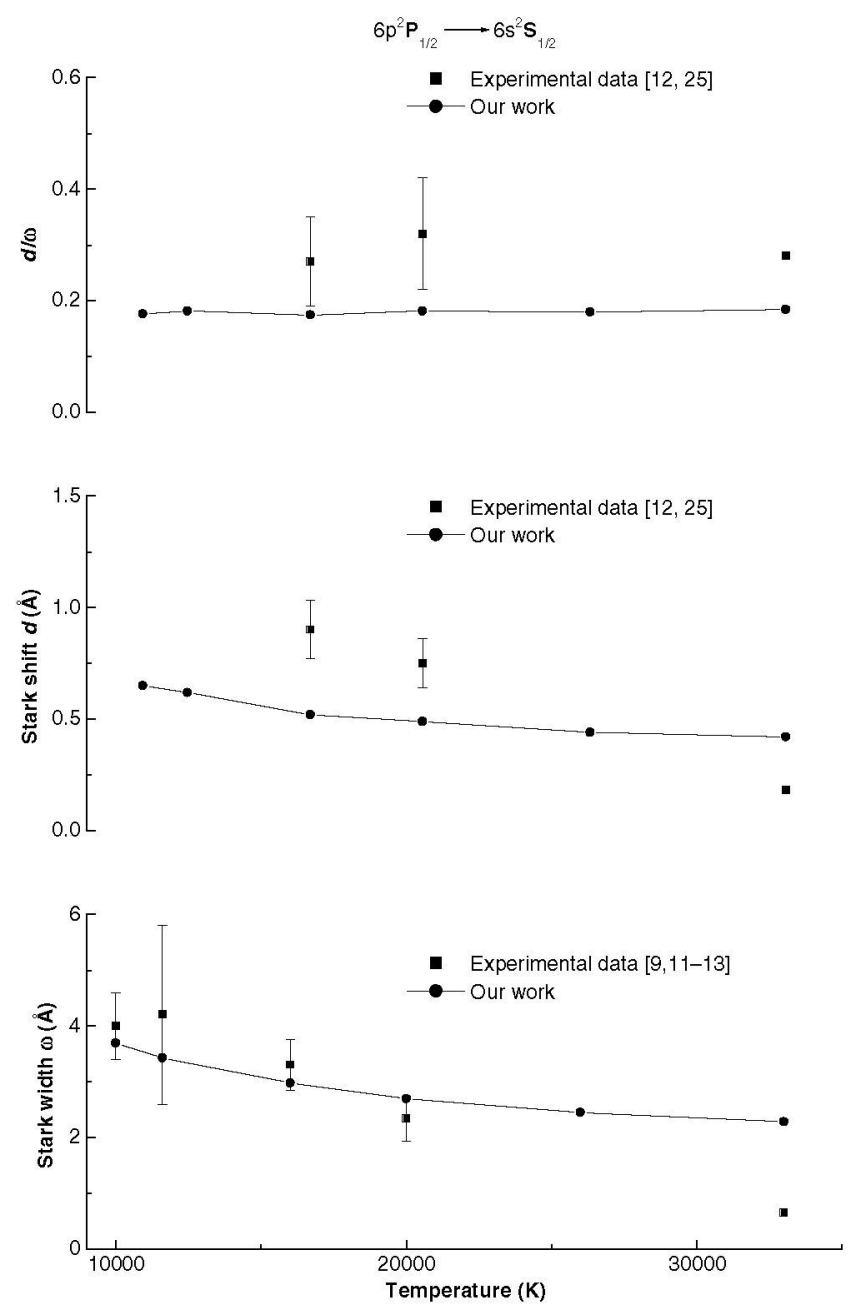

Figure 2. Measured and calculated Stark widths FWHM $(\omega)$, Stark line shift $(d)$ and $d / \omega$ ratios versus temperature for $6845.4 \AA$ Sn II line.

are found to be 2.5 times smaller compared with those other authors [26]).

In table 3 , Stark widths of transitions arising from $n \mathrm{~d}$ ${ }^{2} \mathrm{D}_{3 / 2,5 / 2}(n=5-10)$ to $m p{ }^{2} \mathrm{P}_{1 / 2,3 / 2}(m=5,6)$ are displayed. For these transitions our results are again in good agreement with the existing experimental data. The Stark width values that present an asterisk have been calculated using the approximation suggested by Griem for lines with a nearby perturbing level with allowed dipole transition dominating in the sums of equation (1).

In table 4 , we present results for transitions arising from the levels $n f{ }^{2} \mathrm{~F}_{5 / 2,7 / 2}(n=4,5)$ levels to $5 \mathrm{~d}^{2} \mathrm{D}_{3 / 2,5 / 2}$ and $5 p^{2}{ }^{2} \mathrm{D}_{3 / 2,5 / 2}$. For these transitions our results are in good agreement with the existing experimental data (with the exception already commented of Djenize [12]).

In table 5, we present results for several experimental line shifts normalized at $N_{e}=10^{17} \mathrm{~cm}^{-3}$ and at several temperatures. As is well known, both positive and negative contributions of perturber level can be present in Stark lineshifts calculations [14]. Sometimes near cancellation occurs, resulting in very small shifts for lines which have large widths. In these cases, the experimental shift values can be smaller than the uncertainty of any semi-classical calculation. We can be expect discrepancies with experimental values 
where relatively large configuration mixing is present. Also in this case an approximate factor 2.5 in the measurements of Djenize with regard to those other authors is present $[12,26]$. Judged in this basis we concluded that our shift calculations are successful.

In figure 2, we present measured and calculated Stark widths FWHM $(\omega)$, Stark line-shift $(\boldsymbol{d})$ and $\boldsymbol{d} / \omega$ ratios versus temperature for $6845.4 \AA$ Sn II line. Good agreement between our calculations and the experimental results can be expected because no appreciable configuration mix is present in this case.

Comparison between our values and the experimental values has been possible in all cases because the weight of other broadening mechanisms (ions contributions, ...) can be rejected under the conditions of the different experimental consulted works [27].

\section{Conclusions}

We report the calculated values of the Stark widths for 80 lines (experimental information exists for 23 lines) and Stark shifts for 5 lines of Sn II using the semi-classical approximation.

The contribution of this work consists of taking into account in the calculations the autoionization levels not previously introduced by other authors.

Data are presented for an electron density of $10^{17} \mathrm{~cm}^{-3}$ and several temperatures. Our results are compared with available experimental data. A reasonable agreement between our values and the experimental values has been found as can be seen in figure 2. As can be observed, clear trends in the Stark widths are seen in our results.

\section{Acknowledgment}

The authors acknowledge financial support from the Spanish Universidad Politécnica de Madrid (UPM) project Ref. A 0401. 